\title{
aTUG: Fully-automated Timed Up and Go Assessment Using Ambient Sensor Technologies
}

\author{
Thomas Frenken*, Björn Vester*, Melina Brell*, Andreas Hein* \\ ${ }^{*}$ OFFIS - Institute for Information Technology, Escherweg 2, D-26121 Oldenburg, Germany, Email: thomas.frenken@offis.de
}

\begin{abstract}
A novel approach to fully automating the Timed Up \& Go (TUG) assessment test in professional and domestic environments is presented. The approach, called aTUG, is based on the usage of ambient sensor technologies i.e. two light barriers, four force sensors, and a laser range scanner built into a single apparatus i.e. a chair. aTUG supports execution and documentation of traditional TUG and enhanced component-based TUG. aTUG defines five components: Standing up, walking there, turning, walking back, and sitting down. Algorithms for detection of those components and for computation of their duration and the duration of the whole TUG are presented. An experiment with five elderly patients aged 74-91 years, four female, one male, most multi-morbid has been conducted in a residential care facility in Oldenburg, Germany. Results of the experiment show that aTUG can reliably and precisely measure total duration of TUG and durations of the single components with mean error of only 0.05 seconds and mean standard deviation of 0.59 seconds using especially its force and range measurements. German patent is pending for the presented approach.
\end{abstract}

Index Terms-aTUG, Timed Up and Go (TUG), Mobility Assessment, Laser Range Scanner, LIDAR, Force Sensors, Sensor Fusion

\section{INTRODUCTION}

The so called double aging of the society is a result of the demographic change. It leads to more elderly people requiring health services and less young people financing and providing these services. Another problem of high age is that prevalence of many diseases increases. In Germany, the number of people suffering from at least two diseases increases every 15 years by $20 \%$ starting at the age of $40-54$ years. While in this age group only about $40 \%$ of all people are multi-morbid, this percentage increases to as much as $80 \%$ in people aged 70-85 years [1]. The branch of medicine which deals predominantly with elderly and multi-morbid patients is called geriatrics. One problem about multi-morbidity is that an exact diagnosis often becomes hard to make due to interchange-effects of the various diseases. Therefore, the ultimate aim of each geriatric treatment is to recover and maintain an independent lifestyle of the patients. Making an exact diagnosis becomes less important, instead the functional status of patients is estimated and possible deficits are removed by means of rehabilitation or provision of aid. The estimation of a geriatric patient's functional status happens within the geriatric assessment which is a "multidimensional process designed to assess an elderly person's functional ability, physical health, cognitive and mental health, and socio-environmental situation" [2]. In order to assess a person's functional ability in a certain domain various so called assessment tests have been developed. Their reliability and specificity have been proven in clinical studies. In some countries e.g. in Germany certain assessment tests have to be completed with certain scores within hospitals before a patients can be accounted as a geriatric patient towards health insurance companies.

Functional ability to move around and to get into and hold certain body positions i.e. mobility is one of the most important aspects of a geriatric assessment. Mobility is a fundamental requirement for an independent lifestyle. The probably most often applied assessment test in the field of mobility is the so called Timed Up \& Go (TUG) test [3]. Although assessment tests are widely used in daily clinical practice some problems have been found over time. Assessment tests are only infrequently performed and only in unobstructed environments like hospitals or medical practices. Additionally, they are often perceived as test situations which makes patients perform at their best. Assessment results do thus only partially reflect the real performance of patients which they can call up in daily life at home. Subjective execution by caregivers makes comparison of results among patients or even of results from different executions difficult. Due to missing technical support, assessment results are often still documented manually, which makes their execution time-intensive and personnel-intensive. Such problems are even intensified by the demographic change since there will be more geriatric patients in the future and probably less people to supervise the assessment tests. This means that there is a clear need for support in making execution of assessment tests in daily clinical practice more effective. By bringing assessments to the domestic domain, their potential could be exploited for identifying problems as early as possible and even after a patient has been checked out.

Within this paper a novel approach to supporting the execution and documentation of the TUG assessment test by use of exclusively ambient sensor technologies is presented. We call this new approach ambient Timed Up \& Go (aTUG). This paper introduces our new approach and proves its reliability and precision in execution of TUG and component-based TUG (section II-B). A medical motivation for supporting the TUG assessment technically and an overview of the state-of-the-art in the field of technically-supported TUG is given. Results of an experiment with five elderly residents aged 74-91 years conducted in a residential care facility are presented. 


\section{Medical Motivation}

A geriatric treatment's main aim is to recover and maintain an independent lifestyle of a patient. Due to multi-morbidity a detailed diagnosis is often not possible. Therefore, the geriatric assessment process focuses on the estimation of remaining functional abilities so that deficits hindering an independent lifestyle can be removed. Mobility is one of the most important domains since it is a fundamental requirement for an independent lifestyle. Functional abilities are assessed using various assessment tools. Within the field of mobility the TUG test [3] is the probably most frequently used one. Within this chapter the use of the TUG assessment test is briefly motivated and its limitations are shown.

\section{A. Timed Up and Go}

The Timed Up \& Go has been introduced by D. Podsiadlo and S. Richardson in 1991 [3] as a simplified clinical test for evaluating, especially elderly, patients' general mobility and balance. Within TUG a stopwatch is used to measure the time a patient takes to complete a set of components: rise from a chair, walk $3 \mathrm{~m}$, turn around, walk back, and sit down again. The TUG test thus includes many different mobility skills such as standing-up, sitting-down, turning around, and walking straightly. Measuring only the time to complete the test was meant to objectify a previous version of the test in which performance of patients was graded on a rather subjective 5point scale [4]. The test starts upon a caregiver's signal and the patient is meant to walk at his or her normal speed and to make contact with the chair's back before starting and in order to complete the test. Use of walking aids and armrests is allowed. According to the time taken by the patient to complete the test, he or she is arranged into a result group which gives a hint to the treating caregiver for required actions.

TUG has good test-retest reliability [5]. The test reflects balance deficits [6]. A set of subjects without balance deficits was found to complete the test in under ten seconds whereas a sample with impaired mobility skills and problems in performing activities of daily living (according to the Barthel Index) took more than 30 seconds [7]. TUG can also be used to predict risk of falling in the elderly population [8]. TUG was frequently used in the field of Parkinson's Disease (PD) to distinguish patients in moderate-to-severe state of the disease from healthy control subjects [9].

\section{B. Limitations}

Despite its use in every-day clinical practice and various studies, TUG is limited. TUG was designed to have a simplified and objective outcome measure. It thus focuses only on the time used by the patients to complete the whole test. Time taken to perform the single components of the test and other deficits of moving are ignored. Therefore, Wall et al. [10] proposed the so called Expanded Timed Get-up-and-Go (ETGUG). In ETGUG the durations of all components of TUG are measured separately using a multimemory stopwatch. Additionally, they proposed to extend the walking distance from three to ten meters to allow for better delineation of the component phases. Results of TUG are also sensitive to usage of assistive devices and different types of chairs especially regarding availability of armrests. Medley and Thompson have shown that TUG results vary with usage of assistive devices and that results should not be compared if the device changed [11]. Although the description of TUG is standardized, execution of the test is different in many aspects among institutions. The turning e.g. may either be performed on the spot or patients may walk in a curve especially when using a walking frame. Additionally, the distance patients are meant to walk varies among institutions and executing caregiver. Such subjective execution makes results among patients hard to compare.

Despite those limitations specific to TUG, some general problems about current usage of assessment tests have been identified [12] e.g. place of execution, test awareness of patients, subjective execution by caregivers, and required effort for executing and documenting assessment tests.

\section{STATE OF THE ART}

Today, assessment of mobility is mainly executed in professional care facilities like hospitals or rehabilitation facilities. In case of severe injuries or problems with prostheses or implants laboratories equipped with camera-based systems for cinematic gait analysis based on marker tracking, fluoroscopy systems, systems for cinetic gait analysis of ground reaction forces utilizing force platforms, and dynamic electromyography may be utilized. Recent research investigated mobility (tele-)monitoring especially in the home of affected people. Two main approaches arose using either wearable sensors or sensors installed into the environment. In the field of technical support for clinical assessment tests only very little research has been conducted so far. To the knowledge of the authors, only two papers describing original research to supporting the TUG technically have been published so far.

\section{A. Technical TUG}

Technical support of TUG has so far only been described by use of inertial sensors i.e. gyroscopes and accelerometers. Higashi et al. [13] have investigated the detection of the single components of TUG by using two gyroscopes and accelerometers attached to the subjects' waists and lower limbs. The technically-supported version of TUG was called TUG-T. Based on data retrieved from healthy subjects, characteristic features regarding 3D acceleration and angular velocity identifying the six components (standing-up, walking forward, turn 1, walking backward, turn 2, sitting down) of TUG-T were found. 20 hemiplegic patients, ten able to walk independently, the other ten only with supervision, took part in an experiment. After the experiment, therapists measured the duration of the components from video. A good correlation $(r=0.998)$ was found between the total time measured by the proposed method and the time measured by the therapists.

Recently, Salarin and Zampieri published a series of papers on an instrumented version of TUG called iTUG [14] and its application to the field of mobility assessment in PD 
patients in inpatient situations [15] and at home [16]. Seven inertial sensors attached on the forearms (two 2D gyroscope), shanks (two single axis gyroscopes), thighs (twp single axis gyroscopes), and the sternum (combined 3D accelerometer and $2 \mathrm{D}$ gyroscope) were used to measure the time of the four major components of iTUG: sit-to-stand, steady-state gait, turning, and turn-to-sit. Additionally, a huge set of outcome parameters regarding balance and gait was calculated for each component. In difference to TUG, iTUG uses a seven meter walking distance and an armless chair. Significant differences between early PD patients and age-matched controls groups where found in all components of iTUG except sit-to-stand. Total time of iTUG was not sensitive enough to separate the groups. Test-retest reliability was good. Gait initiation and termination have not been fully explored and might provide more interesting information about patients' health status.

\section{B. Limitations}

In every-day clinical practice, the TUG assessment test may be very well instrumented by using body-worn sensors. Researchers have shown that results are reliable. However, the need to attach body-worn sensors to the patients may increase time-effort used for performing the test compared to using only a stop-watch. For some sensors, required calibration may take even more time. Additionally, handling such devices often requires an expert. Even health care professionals might not be able to correctly handle them. Assessments are often perceived as test situations in hospitals which can make results less reliable. Explicitly donning body-worn sensors before the test will even worsen the situation for the patients. Regarding a potential application of TUG in domestic environments, body-worn sensors would have to be handled by layman in unsupervised situations. Many people, especially demented ones, will not be able to handle those devices on their own. In domestic environments assessment tests could deliver more reliable results when being executed several times a day. People would have to wear the sensors throughout the whole day, which might be uncomfortable. In summary, bodyworn sensors are suitable for technically supporting TUG in clinical practice, but usage is sometimes complicated and timeintensive. When it comes to supporting TUG in domestic environments, usage of body-worn sensors may pose several problems since unsupervised usage of sensors by layman may be erroneousness and uncomfortable.

\section{APPROACH}

Our novel approach to technically supporting TUG is based on the exclusive use of ambient sensor technologies and data fusion algorithms combined in a single transportable apparatus. We call this approach ambient Timed Up \& Go (aTUG). aTUG is designed to have three expansion stages (Figure 1) which built upon another:

a) Stage 1 - Support for traditional TUG: aTUG will fully automate the execution and documentation of traditional TUG (dark gray box in figure 1). Since the duration value

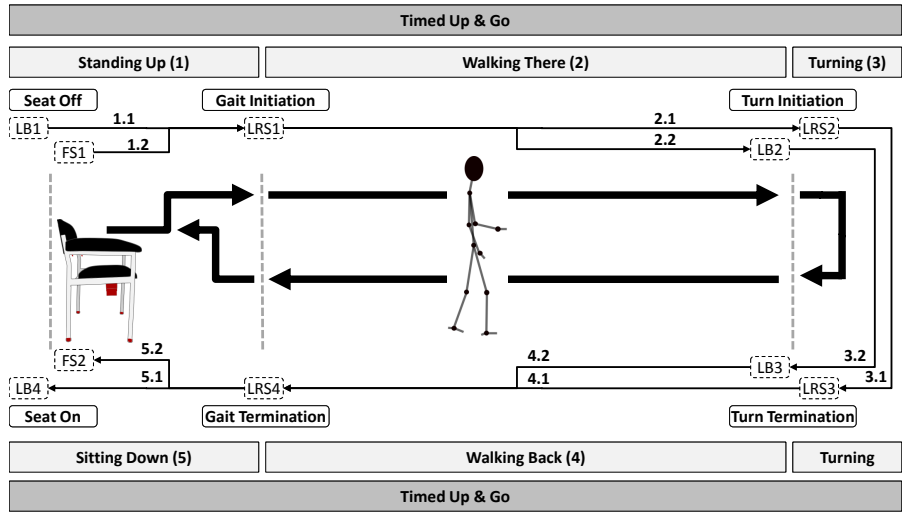

Fig. 1. aTUG Concept and Expansion Stages

will be recorded by objective sensor technologies results will be more reliable and comparison of results will be enabled.

b) Stage 2 - Support for component-based TUG: aTUG will further enhance and objectify TUG by measuring not only the complete duration to finish the test but by also measuring the duration of the single components (light gray boxes in figure 1). aTUG has five components: Standing up, walking there, turning, walking back, and sitting down. For more details on component definition refer to section IV-A.

c) Stage 3 - Gait analysis: aTUG will additionally perform gait analysis by computing a set of spatio-temporal parameters of gait and balance. These parameters will give more detailed insights into mobility of patients and might make aTUG more sensitive to changes in mobility.

First, aTUG will be applied in professional care facilities supporting medical experts in daily clinical practice while executing the TUG assessment test. On a long term, aTUG is also meant to perform TUG in unstructured environments e.g. in peoples' homes or care facilities. This might work supervised e.g. by tele-presence of experts but our final aim is to make TUG executable in an unsupervised and permanent manner. This will enable to exploit TUG's sensitivity to selfcare ability for early detection of problems and for follow-up after patients have been checked out from hospital.

This paper focuses on the two first stages of aTUG. Within this section the concept of aTUG for technically supporting traditional TUG and its enhanced component-based version is described first. Afterwards the two main technical parts of our new approach are explained: the apparatus equipped with various ambient sensors for measuring patients during the test and the sensor processing concepts for computing the outcome of TUG and its components.

\section{A. aTUG vs. TUG: Events and Components}

Within traditional TUG the only outcome measure is the total time taken by the patients to complete the whole test. Within its first stage, aTUG measures exactly this time by processing the data (section IV-C) using the sensors built into the aTUG apparatus (section IV-B). Within its second expansion stage, aTUG also measures the time taken by the 
patients for the single components of TUG (Figure 1). aTUG defines the following components: Standing up (1), walking there (2), turning (3), walking back (4), and sitting down (5). Components in aTUG are defined by an start-event and an end-event. Available events (white solid boxes with rounded edges in figure 1) are "seat off" (lost contact to the back of the chair), "gait initiation" (started to walk away), "turn initiation" (started to turn around), "turn termination" (fully turned towards the chair), "gait termination" (standing in front of the chair again), and "seat on" (made contact to back of the chair again). Those events are detected objectively by processing the available sensor data (section IV-C). The time between those events corresponds to the duration of the components of TUG e.g. the duration of "walking there" is computed by subtracting the time the event "turn initiation" occurred by the time "gait initiation" happened. Some events have alternative event definitions due to more than one sensor being capable of detecting the event (white dashed boxes with rounded edges in figure 1). Figure 1 shows the stages of aTUG, its components, events, and event definitions.

\section{B. aTUG Apparatus}

aTUG aims at supporting TUG in various environments i.e. professional care facilities as well as in domestic environments of patients. Therefore, it has to be easy to make required sensors available in those environments and ideally to remove those if no longer required. A chair is always required for performing TUG. Therefore, aTUG is based on the idea to integrate various ambient sensor technologies into a chair. This makes it possible to move the apparatus to different rooms or change its orientation in home environments, without affecting the results. Two prototypes were developed using different models of chairs to allow ambient measurements in clinical and home environments. While the clinical prototype fulfills all requirements for daily clinical use (safety for patients and hygienic issues), the other prototype enables the apparatus to blend well with domestic environments for measurements at home. Sensors do not necessarily have to be equipped with a chair but might also be placed near or on a chair available in the test environment.

Figure 2 shows the prototype for clinical use. We modified the frame of a chair (A) originally used for blood withdrawal to contain four force sensors (B) based on strain gauge technology. This allows to measure the weight distribution of a person sitting on the chair or when using armrests. An infrared light barrier respectively its sender (C) and receiver (D) are located under the armrests to detect contact to the back of the chair. This solution was used instead of i.e. pressure mats, because no minimum force is required to trigger the sensor. The control box (E) is located under the seat and contains the amplifiers for the pressure sensors, a microcontroller board for signal processing and controlling the light barrier, as well as the power supply and a laser range scanner (LRS). The casing protects the equipment, while the measurements by the LRS can be conducted through an opening in the front. The data is transfered to a PC using USB. An optional light barrier on

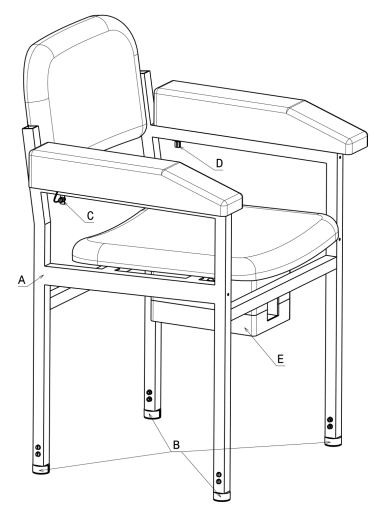

Fig. 2. aTUG Clinical Apparatus Prototype

a wooden bracket can be used to measure the reaching of the end of the straight walking path.

\section{C. aTUG Sensor Processing}

Sensor data collected by the available sensors are preprocessed by a microcontroller board and transferred via USB to a connected PC. Collected information is processed in order to detect the defined events (Figure 1) which are then used to compute the total duration of TUG and the duration of its components (section IV-C4). Some events can be detected by more than one sensor's data and thus those events have more than one possible definition. This section describes how the data collected by the available sensors is processed in order to detect the defined events and how detected events are used to compute the duration of the aTUG components. The aTUG apparatus has the following sensors: Two light barriers $L_{1}$ and $L_{2}$, four force sensors $F_{1 . .4}$, and one laser range scanner $L S_{1}$. Sensors deliver scan data every scan interval $k$. The measurements start at $k_{0}$, the total time of a pass is $T_{0} * k$. The following subsections describe the sensor data processing.

1) Event Detection by Light Barriers: The two light barriers $L_{1}$ (back of the chair) and $L_{2}$ (additional light barrier at the end of the walking path) can only detect whether they are currently activated or not. Their scanning functions contact $_{L_{1}}(k)$ and contact $_{L_{2}}(k)$ deliver the value 1 if currently active and 0 if not for each scanning interval $k$. Using these scanning functions four events i.e. $L B 1$ representing "seat off", $L B 2$ representing "turn initiation", $L B 3$ representing "turn termination", and $L B 4$ representing "seat on" can be detected. $L B 1$ is the first activation of $L_{1}$ after a new pass was started, $L B 2$ is the first activation of $L_{2} . L B 2$ and $L B 4$ are the second activations. Events are only detected correctly if they happen in the ascending order $L B 1, L B 2, L B 3$, and $L B 4$. In summary, the event detection $e(k)$ decides on the $L B x$ events in the following way:

$$
e(k)=\left\{\begin{array}{l|l}
\text { LB1 } & \text { contact }_{L_{1}}(k)=1 \\
\text { LB2 } & {\text { LB } 1 \wedge \text { contact }_{L_{2}}(k)=1}_{\text {LB3 }}(k) \wedge \text { contact }_{L_{2}}(k)=1 \\
\text { LB4 } & \text { LB3 } \wedge \text { contact }_{L_{1}}(k)=1
\end{array}\right.
$$




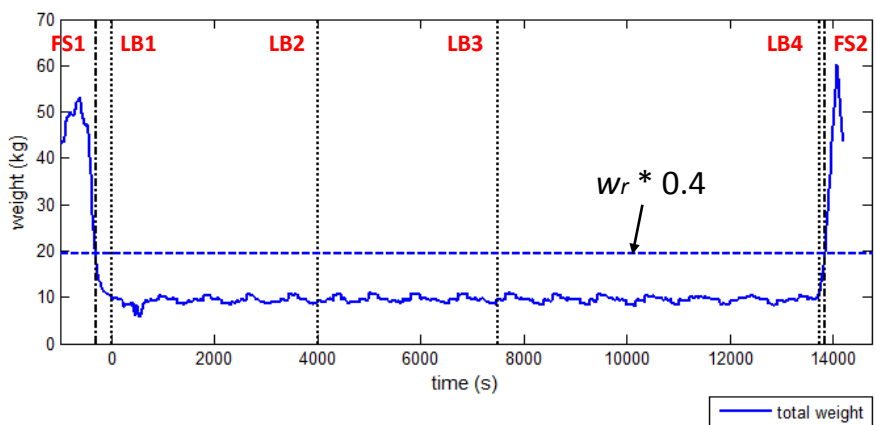

(a) Component Detection Using Force Sensors

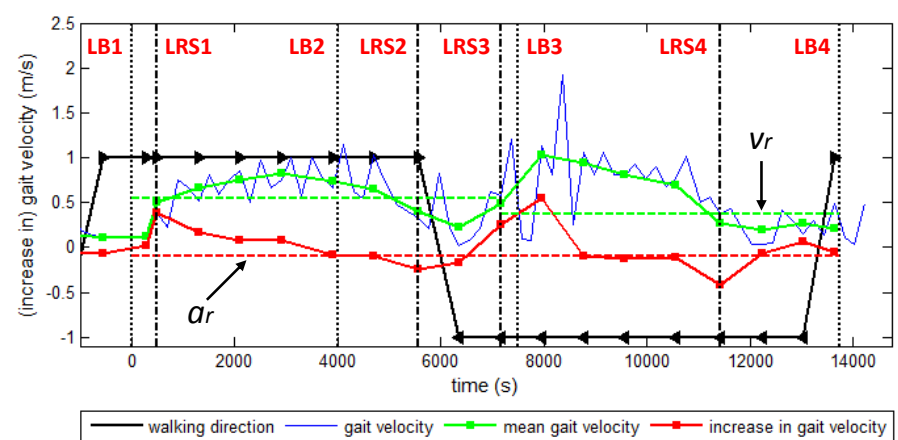

(b) Component Detection Using Laser Range Scanner

Fig. 3. aTUG Sensor Processing for Component Detection

2) Event Detection by Force Sensors: Four force sensors $F_{1 . .4}$ have been integrated into the four legs of the aTUG apparatus. They measure the approximate weight in kilograms on each leg by their scanning functions weight $t_{F_{1,4}}(k)$ for each measurements interval $k$. Currently, event detection of force sensors works exclusively by comparing the total weight of the patient $w_{k}=\sum_{a=1}^{4}$ weight $_{F_{a}}(k)$ to a reference value $w_{r} . w_{r}$ is meant to correspond to the total weight of the patient and is equal to $w_{k_{0}}$. Using the force sensors only two events can be detected i.e. FS1 representing "seat off" and FS2 representing "seat on". $F S 1$ is detected as soon as the total weight $w_{k}$ measured by the force sensors falls below $w_{r} * 0.4$. $F S 2$ corresponds to the first measurement interval $k$, after $F S 1$ was detected, in which $w_{k}>w_{r} * 0.4$. FS1 and $F S 2$ thus represent the times the patient lifts or lowers his or her own weight, respectively $60 \%$ of it, from or to the chair's seat. In summary, the event detection $e(k)$ decides on the $F S x$ events in the following way:

$$
e(k)=\left\{\begin{array}{l|l}
F S 1 & w_{k}<w_{r} * 0.4 \\
F S 2 & F S 1 \wedge w_{k}>w_{r} * 0.4
\end{array}\right.
$$

Figure 3.a shows the force sensors' measurements during a pass and the detected events $F S 1$ and $F S 2$. The total weight $w_{r}$ of the person was approx. 56 kilograms.

3) Event Detection by Laser Range Scanner: A laser range scanner $L S_{1}$ is mounted under the seat of the apparatus scanning into the walking direction of the patient. Its scanning function $\operatorname{scan}(k, \alpha)$ delivers range measurements $r_{k, \alpha}$ for each measurements set $k$ and scan angle $\alpha$ within the scan range of the device $\left[s t a r t_{\alpha}, e n d_{\alpha}\right]$. The event detection works based on our approach to self-selected gait velocity computation presented in [17]. The algorithm presented computes the approximate location of a person's center of mass for each measurement set. Thereby, for each measurement set $k$ the difference vector $\vec{d}_{k}$ to the position of the patient in the previous measurement set $k-1$ and the patient's current gait velocity $v_{k}$ are available. $\vec{d}_{k}=\left(\begin{array}{l}d_{x} \\ d_{y}\end{array}\right)$ is used to compute the movement direction

$$
w d_{k}=\left\{\begin{array}{l|l}
1 & d_{y}>0 \\
-1 & \text { else }
\end{array}\right.
$$

telling whether the patient moves away $(w d(k)=1)$ or towards $(w d(k)=-1)$ the apparatus (black line in Figure 3.b). $v_{k}$ gives the self-selected gait velocity for each measurement set. With $T_{0}=100 \mathrm{~ms}$, which is the scan duration of the laser range scanner, $v_{k}$ is very sensitive to the small changes regarding range measurements in each gait cycle (blue line in Figure 3.b). Since the laser range scanner measures both legs, $v_{k}$ alternates heavily between slow and high values since the scanner measures the patient alternating in stance and swing phase of the gait cycle. During stance phase only very little movement is detected and thus the resulting gait velocity is low. During swing phase the patient moves forward and the resulting $v_{k}$ is high. In order to smoothen this effect, $v m_{k}$ is computed which is the mean gait velocity in $\mathrm{m} / \mathrm{s}$ (green line in Figure 3.b). Additionally, the first derivative $v m_{k}^{\prime}$ is computed which corresponds to the patients acceleration in each measurement set $k$ (red line in Figure 3.b). Using these three values $w d_{k}, v m_{k}$, and $v m_{k}^{\prime}$ four events can be detected: $L R S 1$ representing "gait initiation", $L R S 2$ representing "turn initiation", $L R S 3$ representing "turn termination", and $L R S 4$ representing "gait termination". For all events, the first constraint is the current acceleration. $v m_{k}^{\prime}$ has to be above or below an empirically chosen threshold $a_{r}=-0.1$ (horizontal dashed red line in Figure 3.b). The second constraint is regarding the mean gait velocity $v m_{k}$ which has to be above or below a dynamically computed threshold $v_{r}$ (horizontal dashed green line in Figure 3.b). Initially, $v_{r}$ is set to be $0.35 \mathrm{~m} / \mathrm{s}$ which is a speed even impaired people can reach very fast. During the pass, $v_{r}$ is continuously updated with $v_{r}=\frac{\sum_{i=k}^{k_{0}} v m_{i}}{k-k_{0}}$ as new velocity values are available. The third constraint is the walking direction $w d_{k}$ which is especially used for detection of "turn initiation" and "turn termination". Again, events have to happen in ascending order. In summary, the event detection $e(k)$ decides on the $L R S x$ events in the following way:

$$
e(k)=\left\{\begin{array}{l|l}
L R S 1 & v m_{k}^{\prime}>a_{r} \wedge v m_{k}>v_{r} \\
L R S 2 & v m_{k}^{\prime}<a_{r} \wedge v m_{k}<v_{r} * 0.8 \wedge w d_{k}>0 \\
L R S 3 & v m_{k}^{\prime}>a_{r} \wedge v m_{k}>v_{r} * 0.5 \wedge w d_{k}<0 \\
L R S 4 & v m_{k}^{\prime}<a_{r} \wedge v m_{k}<v_{r} * 0.5
\end{array}\right.
$$

Figure 3.b shows the laser range scanner's measurements during a pass and the detected events $L R S 1, L R S 2, L R S 3$, and $L R S 4$. 
4) Component Duration Computation: All events are detected at a certain point in time $T_{0} * k$. Using these times, the duration of the components of aTUG and the total time can be computed. For some components, alternative event definitions are available since some events can be detected by more than one sensor. Figure 1 visualizes the components of TUG, their corresponding events, and the event definitions described in the previous section. Additionally, the possible computations for the durations of the components are visualized by lines between the event definitions and are named in a unique way. As an example, the line between event definition $L B 1$ and LRS 1 named "1.1" corresponds to the computation of the duration of component "Standing Up" by subtracting the point in time $L R S 1$ was detected by the point in time $L B 1$ happened. The line "1.2" is an alternative definition of this duration by using the events $F S 1$ and $L R S 1$.

\section{EXPERIMENT}

Besides proofing the general feasibility of aTUG to automate TUG, the conducted experiment had two main goals: To compare the precision of video-based measurements, stopwatch measurements, and sensor measurements in traditional TUG and to compare the precision of the different sensors (1), and to evaluate whether the aTUG apparatus and algorithms can be used to reliably compute the duration of the aTUG components compared to video recordings (2). During this experiment a sensors is considered to be "precise" according to how exactly and reliably it enables the computation of the duration in seconds of TUG and its phases.

The experiment was conducted with five elderly patients, four female and one male, with age ranging from 74 to 91 years in a residential care facility in Oldenburg, Germany. All patients suffered from age related medical conditions, which were documented. Most patients were multi-morbid. However, the aim was not to compare different patients' assessment results to each other or the impact of their medical conditions to the assessment results, but to compare the accuracy of time measurement. The patients were allowed to use the armrests of the chair as well as walking aids, like canes.

Figure 4 shows the experimental setup within an empty flat of the residential care facility. The chair (A), described in section IV-B, stood at the wall and white markings were placed on the floor (B) every half meter as reference marks for video comparison. A second light barrier (E) was placed three meters ahead of the chair in order to mark the end of the three meter walk path. The white marking (D) at the end of the path is four meters in front of the chair and marks the latest turning point. The patients were told to turn between the second light barrier and the four meter marking. We used a cost effective Hokuyo URG-04LX-UG01 laser range scanner with a nominal range of 5.6 meters and a measurement range of $240^{\circ}\left(120^{\circ}\right.$ to each side of the chair). The participants were asked to wear white cuffs $(\mathrm{C})$ to achieve better reflection and thus optimal measurements results with the laser range scanner.

Besides the test setup, a computer for data gathering was placed on a table next to the chair and a tripod mounted

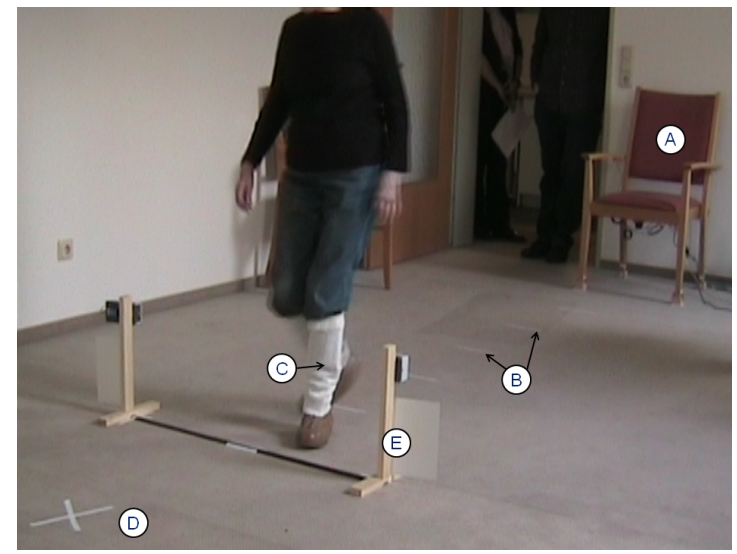

Fig. 4. Experiment Space in a Local Residential Care Facility

video camera documented each pass. The moderator of the experiment was supported by two other persons, a technician for the system and an observer, who measured the timings with a memory stopwatch.

\section{A. Methods}

The experiment was conducted on a single day from 1:30 p.m. until 6:00 p.m. maintaining appropriate light levels. One patient after another was asked into the room, the general goal of the experiment was explained and an informed consent document was signed. The course of actions during the experiment, but no technical details on the measurements, were explained. Additionally, the moderator of the experiment showed the complete procedure himself. Afterwards, the patients were allowed one try to practice the TUG. After a short break, the patients were asked to do a first pass of the TUG. The experiments started after a clear sign of the moderator, measurements started as soon as the patients broke contact with the chair's back. Each attempt was documented by the video camera. A second pass was conducted after another short break. After the experiment, all other questions of the patients regarding technical issues and possible impact for health care were discussed.

\section{B. Results}

Five patients completed aTUG two times each (Table I and II, first column contains number of patient and pass). The total time for TUG was recorded using the sensors and a stopwatch. Additionally, the duration of each component was recorded by using the available sensors. All passes have been recorded by video. Total and components' durations were extracted by a manual video analysis later on. Results of the video analysis are assumed to be most precise (gold standard).

The aim of the first part of the experiment was to compare precision of the stopwatch measurements and the durations computed by using the sensors with the durations from the video analysis. Table I shows the results from the first part of the experiment. Durations in seconds from the video analysis, from the stopwatch measurements, and from using only events generated by light barriers (time between events $L B 4$ and 
TABLE II

Seconds for Completing ATUG Components Using DifFerent MEASUREMEnt TeChniQues

\begin{tabular}{|c|c|c|c|c|c|c|c|c|c|c|c|c|c|c|c|}
\hline & \multicolumn{3}{|c|}{ Standing up } & \multicolumn{3}{|c|}{ Walking There } & \multicolumn{3}{|c|}{ Turning } & \multicolumn{3}{|c|}{ Walking Back } & \multicolumn{3}{|c|}{ Sitting Down } \\
\hline \#, \# & Video & 1.1 & 1.2 & Video & 2.1 & 2.2 & Video & 3.1 & 3.2 & Video & 4.1 & 4.2 & Video & 5.1 & 5.2 \\
\hline 1,1 & 1.32 & 0.50 & $\overline{0.80}$ & 4.64 & 5.07 & 3.50 & 1.79 & 1.60 & 3.50 & 4.51 & 4.27 & 3.93 & 2.43 & 2.32 & 2.41 \\
\hline 1,2 & 1.32 & 1.13 & 1.42 & 4.26 & 2.86 & 2.15 & 1.02 & 1.80 & 2.50 & 4.29 & 4.47 & 4.48 & 0.98 & 1.01 & 0.93 \\
\hline 2,1 & 1.61 & 1.01 & 1.03 & 4.26 & 4.07 & 2.89 & 1.39 & 1.86 & 2.51 & 4.26 & 4.21 & 4.74 & 2.03 & 1.81 & 1.70 \\
\hline 2,2 & 1.89 & 1.12 & 0.98 & 4.51 & 3.66 & 3.30 & 1.85 & 0.80 & 2.75 & 4.76 & 6.26 & 4.68 & 1.89 & 1.66 & 1.70 \\
\hline 3,1 & 1.82 & 0.33 & 0.42 & 4.57 & 4.27 & 3.59 & 1.92 & 2.50 & 2.99 & 4.23 & 4.26 & 4.45 & 1.23 & 1.27 & 1.15 \\
\hline 3,2 & 1.79 & 1.43 & 1.86 & 5.60 & 5.88 & 3.71 & 3.08 & 2.46 & 4.75 & 5.45 & 6.06 & 5.93 & 0.63 & 0.43 & 0.68 \\
\hline 4,1 & 1.69 & 0.42 & 0.73 & 5.73 & 6.06 & 4.35 & 2.23 & 2.43 & 4.24 & 5.14 & 4.86 & 4.76 & 1.41 & 1.50 & 1.49 \\
\hline 4,2 & 1.51 & 1.17 & 1.48 & 5.48 & 6.05 & 3.39 & 1.70 & 0.84 & 3.50 & 5.70 & 5.87 & 5.86 & 0.91 & 0.75 & 0.89 \\
\hline 5,1 & 2.36 & 2.31 & 2.49 & 6.10 & 5.06 & 3.96 & 2.23 & 2.42 & 4.00 & 5.95 & 6.32 & 5.84 & 3.19 & 2.90 & 3.11 \\
\hline 5,2 & 4.32 & 3.56 & 3.98 & 6.20 & 6.46 & 4.63 & 1.95 & 1.67 & 3.81 & 5.42 & 8.36 & 8.05 & 1.14 & 0.94 & 1.18 \\
\hline Mea & ifference & 0.67 & 0.44 & & 0.19 & 1.59 & & 0.08 & -1.54 & & -0.52 & -0.30 & & 0.13 & 0.06 \\
\hline Std & eviation & 0.46 & 0.53 & & 0.69 & 0.44 & & 0.63 & 0.38 & & 0.99 & 0.89 & & 0.13 & 0.12 \\
\hline
\end{tabular}

$L B 1$ ), force sensors (time between events $F S 2$ and $F S 1$ ), and laser range scanner (time between events $L R S 4$ and $L R S 1$ ) are shown. For each measurement method mean difference and standard deviation of the mean difference compared to the video analysis have been computed. Results show that all method except those based on the laser range scanner measurements are highly precise. Mean difference for all methods except LRS are below 1.0s. Standard deviation for measurements based on sensors are slightly lower $(0.33-0.46)$ than for the stopwatch measurements $(0.72)$. This may be due to the fact that sensors measure more objectively than any human. Measurements from LRS are not feasible for computing total time of TUG on their own. This is due to the fact that the LRS can not detect standing up from the chair and thus can start their measurements with the beginning of the second component earliest. In summary, the first part of the experiment has shown that aTUG is capable of precisely automating the execution and documentation of TUG. Measurements by light barriers and force sensors are equally precise as stopwatch measurements. LRS measurements are not feasible for measuring total duration of TUG on their own. The aim of the second part of the experiment was to compare the precision of different sensors or sensor combinations of the aTUG apparatus for measuring the duration of the aTUG components compared to the video results. Table II shows the results of the durations for the various components of aTUG using the video analysis and various sensor combinations (numbers refer to the line names in figure 1). Again, mean difference for all sensor measurements compared to the video results have been computed for all components separately. Standard deviation for the mean difference has been computed as well. Results show that the sensors are capable of computing the duration of all components of aTUG precisely. Mean difference across all components (using the lowest error for each component) is between -0.52 and 0.67 , so below one second. Standard deviation is between 0.12 and 0.89 , also below one second. The highest errors are for measurements regarding "Turning" using light barriers. This is because the
TABLE I

Seconds for Completing TUG Using Different MEASUREMENT TECHNIQUES

\begin{tabular}{|c|c|c|c|c|c|}
\hline$\#, \#$ & Video & Stopwatch & LB4-LB1 & FS2-FS1 & LRS4-LRS1 \\
\hline \hline 1,1 & 14.94 & 15.51 & 13.75 & 14.14 & 10.93 \\
\hline 1,2 & 12.45 & 12.86 & 11.26 & 11.47 & 9.13 \\
\hline 2,1 & 13.61 & 13.80 & 12.96 & 12.87 & 10.14 \\
\hline 2,2 & 13.98 & 14.90 & 13.50 & 13.40 & 10.72 \\
\hline 3,1 & 13.73 & 13.80 & 12.62 & 12.60 & 11.03 \\
\hline 3,2 & 16.54 & 15.88 & 16.25 & 16.93 & 14.39 \\
\hline 4,1 & 16.32 & 15.50 & 15.26 & 15.57 & 13.35 \\
\hline 4,2 & 15.23 & 16.80 & 14.67 & 15.12 & 12.75 \\
\hline 5,1 & 19.60 & 20.40 & 19.01 & 19.40 & 13.80 \\
\hline 5,2 & 21.92 & 22.60 & 20.99 & 21.64 & 16.49 \\
\hline \hline \multicolumn{2}{|c|}{ Mean Difference } & -0.37 & 0.80 & 0.52 & 3.56 \\
\hline \multicolumn{2}{|c|}{ Std. Deviation } & 0.72 & 0.33 & 0.46 & 1.21 \\
\hline
\end{tabular}

light barrier at the end of the three meter path did not directly detect the beginning of the turning but just the time the patients were meant to turn. Most patients walked clearly behind the light barrier and afterwards started to turn. Measurements using the laser range scanner regarding the "Turning" are very precise again. This is due to the fact that the LRS directly detected the turning by its range measurements and resulting velocity and direction computations. In summary, aTUG is capable of extreme precisely computing the duration of the components of TUG. Using only force sensors and the laser range scanner the mean error is 0.05 seconds, mean standard deviation is 0.59 seconds. Combining the first light barrier and the laser range scanner gives slightly worser results of 0.09 and 0.59 seconds.

\section{Discussion}

The experiment has shown that a single light barrier at the backrest of a chair may be used to automate TUG. Force sensors provide equally precise results but may be confused by usage of armrests (further experiments required). The LRS, although not capable of supporting TUG on its own, 
may provide additional information e.g. if the patient walked the full distance. Component-based TUG can be performed combining the LRS with either light barriers or force sensors. The number of patients was rather low in the experiment. Thresholds e.g. for gait velocity $\left(v_{r}\right)$, acceleration $\left(a_{r}\right)$, and weight $\left(w_{r}\right)$ are currently more or less statically defined and based on experience. We want to work on the dynamic refinement of these thresholds according to the patients' individual capabilities in order to make the computation more reliable in larger sets of patients. The algorithms for gait velocity computation presented in [17] and used within this paper will be further enhanced to separate the single legs of the patient. Force sensor data processing is rather simple at the moment, much more information about the patients' balance can be retrieved. Usage of armrests or falling into the chair due to muscle power problems could be detected. In the future, we hope to make aTUG usable for ambient assessments in domestic environments without explicit instructions. For use in home environments, assignment of individual data to different residents has to be achieved by the system itself. Promising solutions seem to be the use of individual weight or gait parameters to differentiate between individuals. We also want to investigate placement of the LRS in different positions next to the chair.

\section{CONCLUSION}

Within this paper we have presented aTUG, a fully automated Timed Up \& Go (TUG) assessment test using ambient sensor technologies. aTUG has three expansion stages: Support in execution and documentation of traditional TUG (1), support of enhanced component-based TUG (2), and gait analysis within TUG (3). Ambient sensors i.e. two light barriers, four force sensors, and a laser range scanner are combined in a single transportable apparatus and algorithms for required sensor precessing have been presented. aTUG is mainly meant to support medical professionals in daily clinical practice but on a long-term also aims at bringing the TUG assessment test to domestic environments. An experiment with five elderly patients aged 74-91 years has been conducted in a residential care facility in Oldenburg, Germany. Results of the experiment show that aTUG can reliably measure total duration of TUG and durations of the single components (standing up, walking there, turning, walking back, and sitting down) using especially its force and range measurements. Although results are precise regarding the computation of TUG phase durations, overall validation against a clinically validated reference system still has to be done.

Future work will be to conduct a clinical trial (ethics votum pending) and to further enhance the algorithms for sensor processing. Especially thresholds used for component detection will be further enhanced by computing individual values during the assessments. Additionally, algorithms for detailed gait and balance analysis (stage 3 of aTUG) will be developed for computing several spatio-temporal parameters of gait and balance (especially during the turning and seat off/on phases).
German patent is pending for the presented approach to automatically conducting TUG using ambient sensors and for automatic gait analysis.

\section{ACKNOWLEDGMENT}

Partially funded by the German Ministry of Eduction and Research within the research project PAGE (01FCO8044).

\section{REFERENCES}

[1] K. Bhm, C. Tesch-Rmer, and T. Ziese, "Gesundheit und Krankheit im Alter," Online, June 2008, ISBN 978-3-89606-196-6.

[2] M. Beers and R. Berkow, Eds., The Merck Manual of Geriatrics. Merck \& Co., Inc., 2006. [Online]. Available: http://www.merck.com/mkgr/mmg/home.jsp

[3] D. Podsiadlo and S. Richardson, "The timed "Up \& Go": a test of basic functional mobility for frail elderly persons." J Am Geriatr Soc, vol. 39, no. 2, pp. 142-148, Feb 1991.

[4] S. Mathias, U. S. Nayak, and B. Isaacs, "Balance in elderly patients: the "get-up and go" test." Arch Phys Med Rehabil, vol. 67, no. 6, pp. 387-389, Jun 1986.

[5] P. Botolfsen, J. L. Helbostad, R. Moe-Nilssen, and J. C. Wall, "Reliability and concurrent validity of the Expanded Timed Up-andGo test in older people with impaired mobility." Physiother Res Int, vol. 13, no. 2, pp. 94-106, Jun 2008. [Online]. Available: http://dx.doi.org/10.1002/pri.394

[6] S. L. Whitney, J. L. Poole, and S. P. Cass, "A review of balance instruments for older adults." Am J Occup Ther, vol. 52, no. 8, pp. 666-671, Sep 1998.

[7] F. I. Mahoney and D. W. Barthel, "Functional Evaluation: The Barthel Index," Md State Med J, vol. 14, pp. 61-65, Feb 1965.

[8] J. Large, N. Gan, D. Basic, and N. Jennings, "Using the timed up and go test to stratify elderly inpatients at risk of falls." Clin Rehabil, vol. 20, no. 5, pp. 421-428, May 2006.

[9] S. Morris, M. E. Morris, and R. Iansek, "Reliability of measurements obtained with the Timed "Up \& Go" test in people with Parkinson disease." Phys Ther, vol. 81, no. 2, pp. 810-818, Feb 2001.

[10] J. C. Wall, C. Bell, S. Campbell, and J. Davis, "The Timed Get-up-andGo test revisited: measurement of the component tasks." J Rehabil Res Dev, vol. 37, no. 1, pp. 109-113, 2000.

[11] A. Medley and M. Thompson, "The effect of assistive devices on the performance of community dwelling elderly on the timed up and go test," Issues Aging, vol. 20, pp. 3-7, 1997.

[12] T. Frenken, O. Wilken, and A. Hein, "Technical Approaches to Unobtrusive Geriatric Assessments in Domestic Environments," in Proceedings of the 5th Workshop on Behaviour Monitoring and Interpretation, BMI'10, Karlsruhe, Germany, September 21, 2010, ser. CEUR Workshop Proceedings, H. A. B. Gottfried, Ed., vol. 678. CEUR-WS.org, 2010, pp. 63-74. [Online]. Available: http://sunsite.informatik.rwth-aachen.de/Publications/CEURWS/Vol-678/BMI10-06.pdf

[13] Y. Higashi, K. Yamakoshi, T. Fujimoto, M. Sekine, and T. Tamura, "Quantitative evaluation of movement using the timed up-and-go test," Engineering in Medicine and Biology Magazine, IEEE, vol. 27, no. 4, pp. $38-46,2008$.

[14] A. Salarian, F. B. Horak, C. Zampieri, P. Carlson-Kuhta, J. G. Nutt, and K. Aminian, "iTUG, a sensitive and reliable measure of mobility." IEEE Trans Neural Syst Rehabil Eng, vol. 18, no. 3, pp. 303-310, Jun 2010. [Online]. Available: http://dx.doi.org/10.1109/TNSRE.2010.2047606

[15] C. Zampieri, A. Salarian, P. Carlson-Kuhta, K. Aminian, J. G. Nutt, and F. B. Horak, "The instrumented timed up and go test: potential outcome measure for disease modifying therapies in Parkinson's disease." $J$ Neurol Neurosurg Psychiatry, vol. 81, no. 2, pp. 171-176, Feb 2010. [Online]. Available: http://dx.doi.org/10.1136/jnnp.2009.173740

[16] C. Zampieri, A. Salarian, P. Carlson-Kuhta, J. G. Nutt, and F. B. Horak, "Assessing mobility at home in people with early Parkinson's disease using an instrumented Timed Up and Go test." Parkinsonism Relat Disord, Aug 2010. [Online]. Available: http://dx.doi.org/10.1016/j.parkreldis.2010.08.001

[17] T. Frenken, M. Gövercin, S. Mersmann, and A. Hein, "Precise Assessment of Self-Selected Gait Velocity in Domestic Environments," in Pervasive Computing Technologies for Healthcare (PervasiveHealth). IEEE, 2010, ISBN 978-963-9799-89-9. 